\title{
Rainfall-runoff prediction using a Gustafson-Kessel clustering based Takagi-Sugeno Fuzzy model
}

\author{
Subhrasankha Dey*1, Tanmoy Dam ${ }^{2}$ Student Member, IEEE
}

\begin{abstract}
A rainfall-runoff model predicts surface runoff either using a physically-based approach or using a systems-based approach. Takagi-Sugeno (TS) Fuzzy models are systems-based approaches and a popular modeling choice for hydrologists in recent decades due to several advantages and improved accuracy in prediction over other existing models. In this paper, we propose a new rainfall-runoff model developed using GustafsonKessel (GK) clustering-based TS Fuzzy model. We present comparative performance measures of GK algorithms with two other clustering algorithms: (i) Fuzzy C-Means (FCM), and (ii) Subtractive Clustering (SC). Our proposed TS Fuzzy model predicts surface runoff using: (i) observed rainfall in a drainage basin and (ii) previously observed precipitation flow in the basin outlet. The proposed model is validated using the rainfall-runoff data collected from the sensors installed on the campus of the Indian Institute of Technology, Kharagpur. The optimal number of rules of the proposed model is obtained by different validation indices. A comparative study of four performance criteria: Root Mean Square Error (RMSE), Coefficient of Efficiency (CE), Volumetric Error (VE), and Correlation Coefficient of Determination (R) have been quantitatively demonstrated for each clustering algorithm.
\end{abstract}

Index Terms-Rainfall-runoff prediction, Takagi Sugeno Fuzzy inference, Gustafson-Kessel clustering, Comparative error measure

\section{INTRODUCTION}

A rainfall-runoff is an overland water flow due to precipitation happening over a drainage basin. A rainfall-runoff model predicts accumulated overland flow at an outlet of a drainage basin due to excess rainfall. A rainfall-runoff model can be developed using either a physically-based model or a data-driven black-box model. A physically-based model is constructed by the mass, momentum, and energy transformation equations such that the parameters of the model are directly related to the characteristics of a drainage basin (or a catchment area) [1]. However, such models ignore frequent topographical changes of a catchment due to urbanization/human interventions and demand additional data (e.g. initial soil moisture, land use, evaporation and infiltration data, distribution, and rainfall duration) to improve model accuracy [2]. In contrast to physically-based approaches, data-driven models can handle the topographic variation of a catchment and can produce output even with the absence of additional parameters and data, those are needed by a physically-based model [3].

*Corresponding author

${ }^{1}$ Subhrasankha Dey, Dept. of Infrastructure Engineering, The University of Melbourne, Parkville, Australia deys@student.unimelb.edu.au

2 Tanmoy Dam, School of Engineering and Information Technology, University of New South Wales Canberra, Australia t.dam@student.adfa.edu.au
Recently, Fuzzy rule-based models are becoming a popular data-driven approach for rainfall-runoff modelling [2], [4], [5], [6], [3]. One of the important Fuzzy rule-based rainfallrunoff models is the Neuro-Fuzzy system, known as Adaptive Network-based Fuzzy Inference System (ANFIS) [7]. ANFIS has been shown superiority when compared with other datadriven models such as Artificial Neural Network (ANN), AutoRegressive Moving Average (ARMA), and Auto-Regressive with exogenous inputs (ARX) models. However, ANFIS is reported to be computationally expensive and tends to be overfitting [8], [3].

Performance of the existing Fuzzy rule-based models varies with the topographic nature of a catchment [3]. Further, the models often suffer from uncertainty in the selection of lagtime, variability of the training data in the presence of many influencing parameters of a hydrological process. Hence, a comparative performance measure is adapted by researchers in order to achieve higher accuracy to predict runoff due to rainfall [2], [9]. Also, Takagi-Sugeno (TS) fuzzy systems remains a popular choice of capturing the non-linear relationships between rainfall and runoff in a catchment [2], [9].

The main contributions of this paper are as follows,

- Gustafson-Kessel's (GK) clustering method has been adapted to learn the fuzzy structure, mainly premise and consequence parameters in TS fuzzy model identification. To obtain the optimal number of rules directly from the data, we used different cluster validating indices.

- To validate the GK-based TS fuzzy model using the data obtained at a catchment area of Indian Institute of Technology, Kharagpur (an academic campus) [10]. We evaluate our proposed model's performance in the multi-step-ahead runoff prediction scheme. We have also compared the performance with other existing clusteringbased Fuzzy methods.

\section{LiterATURE REVIEW}

In earlier research, linear regression-based runoff prediction models are found [11]. However, nonlinear dynamics of runoff due to rainfall were not considered and hence the model performance is not adequate in linear regression. In recent time, the following nonlinear data-driven approaches are becoming popular (i) Artificial Neural Network (ANN) [12], [13], [11] and (ii) Fuzzy rule-based Neuro-Fuzzy Model [4], [5], [6], [14].

The Fuzzy rule-based models are popularly based on the trial and error method to find out the optimal number of rules [11], [15]. In a Fuzzy rule-based approach, membership 
functions and Fuzzy rules are determined with some degree of uncertainty which is present in a hydrological process [3]. An improvement of a Fuzzy rule-based system is found in Neuro-Fuzzy models where a neural network is used to train the model's membership functions and Fuzzy rules [16], [3]. Neuro-Fuzzy systems are often based on Takagi-SugenoKang structure [3]. ANFIS is a widely popular Neuro-Fuzzy system and is used in a number of data-driven rainfall-runoff modelling despite the overfitting issues of finding suitable membership functions [16], [17], [18], [19]. Other studies which have employed Neuro-Fuzzy Models (NFMs) for flow forecasting include DENFIS (Dynamic Evolving Neural-Fuzzy Inference System) models [20]. Wavelet-based approaches are also integrated with the ANFIS model to handle the uncertainty in the observed data for removing the noise samples [21]. The effect of lag time on rainfall-runoff modeling by ANFIS has been studied earlier [22]. Additionally, an input selection method based on correlation and mutual information analysis is able to identify an optimum set of rainfall inputs for rainfall-runoff modeling by ANFIS [22]. Sometimes, nonsequential rainfall antecedents can produce better results compared to sequential rainfall inputs [22]. Hence, selection of the type and number of inputs are also important in a TS Fuzzy rule-based rainfall-runoff model [23]. Rainfalls are considered by some researchers as the only inputs of the data-driven model [24], [25]. When the flow measurement is unavailable for subcatchments in a catchment, rainfall data can be chosen as inputs to identify the more contributing sub-catchments [8]. However, the performance of ANFIS could be affected due to unnecessary complexity when so many inputs are involved [22]. The computational time can be significantly reduced using a fewer number of inputs.

A combination of rainfall and runoff are also used as inputs to the Fuzzy models [15], [26] with increased computation complexity and overfitting errors. Some studies have suggested pruning of the unnecessary inputs by selecting a narrower time window around the most correlated rainfall antecedent with runoff [27], [15]. Hence, in this paper, we develop a simple four-input-based Takagi-Sugeno Fuzzy model to reduce computational complexity and reducing overfitting errors. We present a comparative study of three different Fuzzy clustering algorithms based the proposed TS Fuzzy model using real data collected at a pilot study area of a drainage basin.

\section{General Structure of a TS Fuzzy model}

In TS Fuzzy model, any nonlinear function is approximated by a set of linear models where each linear model is described as a rule [28]. The structure of each rule is represented by premise and consequent variables. An input variable transformed into Fuzzy Membership Function (MF) is known as premise variable, whereas the output of TS Fuzzy model is known as consequent variable. The consequent output is described by a linear functional relation between premise variables. In a TS Fuzzy model, each rule is described as follows,

$$
\begin{aligned}
& P^{i} \text { : IF } x_{k 1} \text { is } A_{1}{ }^{i} \text { AND IF } x_{k 2} \text { is } A_{2}{ }^{i} \text {... AND IF } x_{k n} \text { is } A_{n}{ }^{i} \\
& \text { THEN } y_{k}^{i}=a_{0}^{i}+x_{k 1} \cdot a_{1}^{i}+\ldots+x_{k n} \cdot a_{n}^{i} \\
& =a_{0}{ }^{i}+\sum_{j=1}^{n} x_{k j} \cdot a_{j}{ }^{i}, j=1,2, . ., n, i=1,2, . ., C \\
& =\left[\begin{array}{ll}
1 & x_{k}^{T}
\end{array}\right] \theta^{i}=\bar{x} \theta^{i}
\end{aligned}
$$

where $P^{i}$ is the structure of the rule $(i=1,2, \ldots$, Crule $)$ and $n$ is the number of input variable. $\mathbf{x}_{k}=\left[x_{k 1}, x_{k 2}, \ldots x_{k n}\right]$ be the input variables or premise variables of the model and $y_{k}$ is the consequent's output of $i^{t h}$ rule. $\theta^{i}=\left[p_{0}{ }^{i}, p_{1}{ }^{i}, \ldots, p_{n}{ }^{i}\right] \in$ $R^{(n+1)}$ is the coefficients of consequent variable. The dumbbell shape MF (i.e. Gaussian MF) for each premise variable is considered here as,

$$
\begin{array}{r}
A_{j}{ }^{i}\left(x_{k j}\right)=\exp \left[-\frac{\left(x_{k j}-\vartheta_{j}^{i}\right)^{2}}{\left(\sigma_{j}^{i}\right)^{2}}\right] \in[0,1], \\
\text { where, }(i=1,2, . ., C ; j=1,2, . ., n)
\end{array}
$$

where $V=\left[\vartheta_{1}{ }^{i}, \vartheta_{2}{ }^{i}, \ldots, \vartheta_{n}{ }^{i}\right] \in R^{n}$ is the vector of mean of the GMF. The width is represented by $\left[\sigma_{1}{ }^{i}, \sigma_{2}{ }^{i}, \ldots, \sigma_{n}{ }^{i}\right] \in R^{n}$.

The final output of the TS Fuzzy model is represented by weighted average of each individual firing rule. Let, the system input variables and output variables are, $x_{k}=$ $\left[x_{k 1}, x_{k 2}, \ldots, x_{k n}\right]^{T}\left(k=1,2, \ldots, N^{t h}\right.$ observation $)$ and $y_{k}(k=$ $1, \ldots, N)$ respectively. Therefore, the output of TS Fuzzy model is given by,

$$
\hat{y}_{k}=\frac{\sum_{i=1}^{C} w_{k}{ }^{i} \cdot y_{k}{ }^{i}}{\sum_{i=1}^{C} w_{k}{ }^{i}}
$$

where, the membership value $\left(A_{j}{ }^{i}\left(x_{k j}\right)\right)$ of $j^{t h}$ premise variable of $k^{\text {th }}$ sample is defined in (2). The overall truth value $\left(w_{k}{ }^{i}\right)$ of each rule can be calculated by minimum operator or logic AND operation. The truth value of $k^{t h}$ sample is expressed by,

$$
\begin{array}{r}
w_{k}{ }^{i}=\min _{j}\left\{A_{j}{ }^{i}\left(x_{k j}\right)\right\}, \\
(i=1,2, \ldots, C ; j=1,2, \ldots, n ; k=1,2, \ldots, N)
\end{array}
$$

\section{FUZZY CLUSTERING BASED TS FUZZY MODEL}

\section{A. Fuzzy partition matrix from the model data}

TS Fuzzy model can be designed from the input-output data by Fuzzy partition matrix. Let, the data vector is represented as, $D=\left\{Z_{k}: Z_{k}=\left[x_{k}^{T}, y_{k}\right]^{T}, x_{k}=\left[x_{k 1}, x_{k 2}, \ldots, x_{k n}\right]^{T}, k=\right.$ $1,2, \ldots, N\}$. Hence, the Fuzzy partition matrix in a data set for premise variables is represented by clusters with certain degree of membership values. Each membership value for each cluster is a part of the Fuzzy partition matrix.

Definition Let, $\mathfrak{J}=\left\{Z_{1}, Z_{2}, \ldots, Z_{N}\right\}$ be a finite set and $2 \leq C<N$ be number of clusters, then Fuzzy partition matrix for set $\mathfrak{J}$, is defined as

$$
\begin{aligned}
& U_{f c}=\left\{U \in R^{C \times N} \mid \mu_{i k} \in[0,1] ; \sum_{i=1}^{C} \mu_{i k}=1 ; 0<\right. \\
& \left.\sum_{k=1}^{N} \mu_{i k}<N, \forall i\right\}
\end{aligned}
$$


where, $\mu_{i k}(i=1,2, \ldots, C$ and $k=1,2, \ldots, N)$ be Fuzzy membership value of $Z_{k}$ sample is belonging to $i^{\text {th }}$ cluster.

\section{B. GK clustering algorithm based Fuzzy partition matrix}

The whole input-output space is divided by Fuzzy clustering algorithm to achieve the Fuzzy partition matrix. Therefore, many clustering algorithms based TS Fuzzy models are found in [29], [30], [31]. FCM clustering algorithm is popular of its kind [32]. The modified version of FCM algorithm is known as GK (Gustafson-Kessel) Algorithm [33]. Cluster covariance values of each class is updated by the norm inducing distance metric. The geometrical shape provided by GK clustering algorithm is elliptical shape in nature whereas the FCM gives hyper-spherical shape. The objective function of GK algorithm is given by,

$$
\begin{aligned}
& J_{G K}\left(D ; U, \theta, \mathrm{v}, M_{i}\right)=\sum_{i=1}^{C} \sum_{k=1}^{N} \mu_{i k}{ }^{m} G_{i k}{ }^{2} \\
& \text { subject to, } \sum_{i}^{C} \mu_{i k}=1
\end{aligned}
$$

where the distance metric is $G_{i k}^{2}=\left[Z_{k}-\mathbf{v}^{i}\right]^{T} M_{i}\left[Z_{k}-\mathbf{v}^{i}\right]$, $\mathbf{v}^{i} \in R^{(n+1)}$ is cluster prototype in the input-output product space and $M_{i}$ is symmetric positive definite matrix, represents as volume size of $i^{t h}$ cluster. Equation (5) is a constrained optimization problem. Applying the Lagrangian multipliers $(\lambda)$ to convert it into an unconstrained optimization form,

$$
\begin{aligned}
L(U, \lambda, \theta, \mathrm{v}) & =J_{G K}\left(D ; U, \theta, \mathrm{v}, M_{i}\right)-\sum_{i=1}^{C} \beta_{i}\left(\left|M_{i}\right|-\rho\right) \\
& -\sum_{k=1}^{N} \lambda_{k}\left(\sum_{i=1}^{C} \mu_{i k}-1\right)
\end{aligned}
$$

For minimization of (6) with the respect to $\mu_{i k}$, assuming $G_{i k}{ }^{2}>0$, the final expression is as follows,

$$
\mu_{i k}=\frac{1}{\sum_{q=1}^{C}\left(\frac{G_{i k}{ }^{2}}{G_{q k}{ }^{2}}\right)^{\frac{1}{m-1}}}
$$

Similarly, the cluster center $\left(v^{i}\right)$ is obtained by taking derivative of (5) w.r.t. $\mathbf{v}^{i}$ as,

$$
\begin{aligned}
\frac{\partial L(\ldots)}{\partial \mathrm{v}^{i}}= & \sum_{k=1}^{N} \mu_{i k}{ }^{m}\left[x_{k}-\mathrm{v}^{i}\right]=0 \\
\Rightarrow \mathrm{v}^{i} & =\frac{\sum_{k=1}^{N} \mu_{i k}{ }^{m} Z_{k}}{\sum_{k=1}^{N} \mu_{i k^{m}}}
\end{aligned}
$$

Taking partial derivative (6) w.r.t $M^{i}$,

$$
\begin{gathered}
\frac{\partial L(\ldots)}{\partial M_{i}}=\sum_{k=1}^{N} \mu_{i k}{ }^{m}\left[\mathrm{Z}_{k}-\mathrm{v}^{i}\right]\left[\mathrm{Z}_{k}-\mathrm{v}^{i}\right]^{T} \\
-\beta_{i}\left|M_{i}\right| M_{i}^{-1}=0 \\
\Rightarrow M_{i}^{-1}=\frac{\sum_{k=1}^{N} \mu_{i k}{ }^{m}\left[\mathrm{Z}_{k}-\mathrm{v}^{i}\right]\left[\mathrm{Z}_{k}-\mathrm{v}^{i}\right]^{T}}{\beta_{i}\left|M_{i}\right|} \\
=\sqrt[n]{\left(\frac{1}{\rho\left|F_{i}\right|}\right)} F_{i} .
\end{gathered}
$$

where $F_{i}$ is the covariance matrix. $\rho$ is a constant.

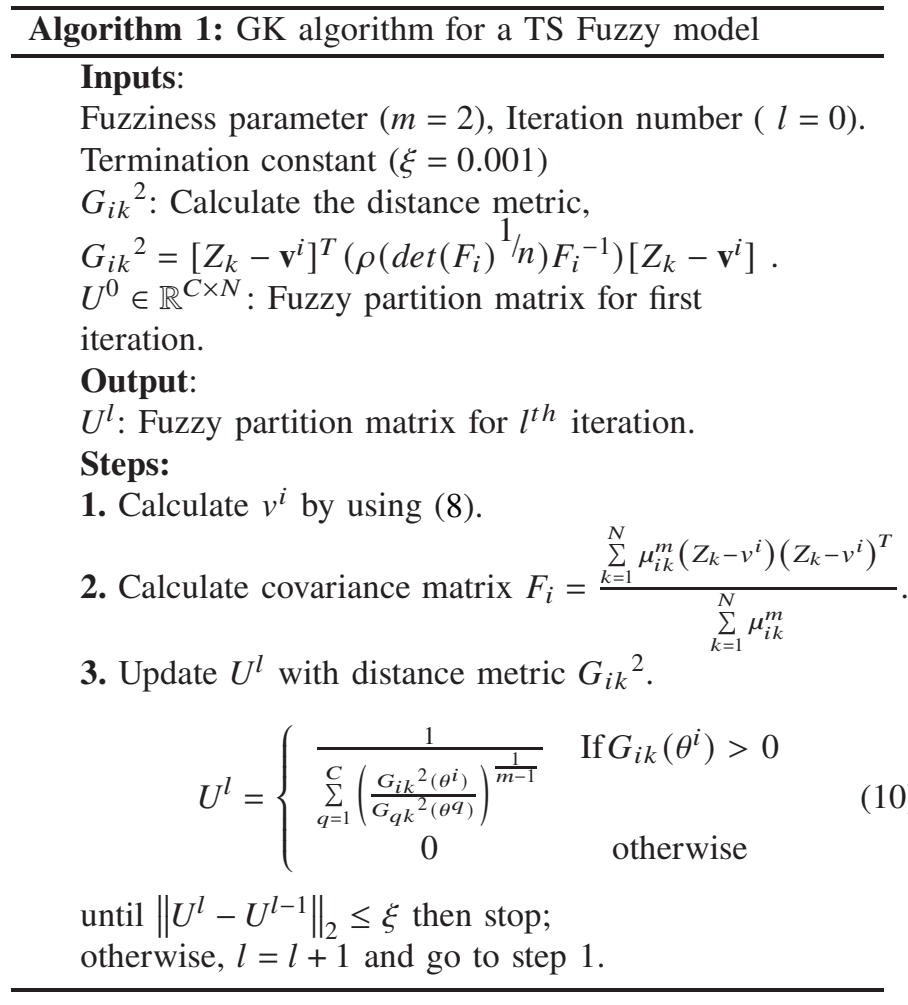

\section{Cluster validity index}

Cluster validity index provides a clear idea about the optimal number of partitions in the data space. The partition in the data space is obtained by clustering algorithms viz. FCM, GK and SC [34]. The optimal number of partitions (subspace) in a data space are obtained by varying the clustering numbers. However, varying the clustering numbers only may not be sufficient enough to provide the optimal numbers due to its dependency in the cluster shape. Therefore, a suitable validity index with proper partitioning algorithm is required to obtain the optimal numbers. Hence, six different validity measures are applied to find out the optimal number of clusters in the data space as follows:

1) Partition Coefficient (PC): The Partition Coefficient (PC) [35]index measures of overlapping between clusters. It is defined by,

$$
V_{P C}=\frac{\sum_{i=1}^{C} \sum_{k=1}^{N}\left(\mu_{i k}\right)^{2}}{N}
$$

where $\mu_{i k}$ is the membership value of $k^{t h}$ data point belonging to $i^{t h}$ cluster. The PC method does not hold any connection between shape of data. It is concerned only with the partition matrix $(U)$ i.e. it does not have any relation with data space. Therefore, this validity index may not favorable for highly complex dataset. The index provides an optimal number of clusters at a point that gives maximum value by varying the cluster number from $C=2$ to $C_{\max }$.

2) Partition Entropy (PE): The Partition Entropy (PE) index [35] defines the fuzziness between partitions and is given 
by as,

$$
V_{P E}=-\frac{\sum_{k=1}^{N} \sum_{i=1}^{C} \mu_{i k} \log \left(\mu_{i k}\right)}{N}
$$

where the minimization of $\left(V_{P E}\right)$ provides the optimal number by varying cluster number from $C=2$ to $C_{\max }$. Similarly, for the $V_{P E}$ index, that may not provide the exact number of partitions in a dataset because it does not hold any relation between the partitions shape.

3) Modified Partition Coefficient (MPC): Both $V_{P C}$ and $V_{P E}$ indices monotonic decreasing tendency with varying $C$. The modified PC (MPC) [36] can remove the monotonic decreasing tendency and is given by as,

$$
\mathrm{V}_{\mathrm{MPC}}=1-\frac{C}{C-1}\left(1-\mathrm{V}_{\mathrm{PC}}\right)
$$

The optimal number of clusters is obtained by maximizing $V_{M P C}$ over the cluster number, varying from $C=2$ to $C_{\text {max }}$.

4) Partition Index (SC): This index [37] is obtained by ratio of the compactness and separation of a cluster. The summation of the individual cluster validity measure is normalized by dividing cardinality of each cluster. The index is given by,

$$
V_{S C}=\sum_{i=1}^{C} \frac{\sum_{k=1}^{N}\left(\mu_{i k}\right)^{2}\left\|Z_{k}-v^{i}\right\|^{2}}{N_{i} \sum_{j=1}^{C}\left\|v^{j}-v^{i}\right\|^{2}}
$$

A lower value of the index indicates the optimal numbers.

5) Separation Index $(S)$ : This index [37] is generally do the opposite effect of SC index. Minimum separation provides the optimal numbers of clusters. It is given by as,

$$
V_{S}=\frac{\sum_{i=1}^{C} \sum_{k=1}^{N}\left(\mu_{i k}\right)^{2}\left\|Z_{k}-v^{i}\right\|^{2}}{N_{i} \min _{i, j}\left\|v^{j}-v^{i}\right\|^{2}}
$$

6) Xie-Beni $(X B)$ : The Xie-Beni index [34] is based on the compactness of the cluster and minimum separation variation between the clusters. The optimal value is obtained from the minimum values of the two ratios by varying the cluster number from $C=2$ to $C_{\max }$. The mathematical expression for $V_{X B}$ is given by,

$$
V_{X B}=\frac{\sum_{i=1}^{C} \sum_{k=1}^{N}\left(\mu_{i k}\right)^{2}\left\|Z_{k}-v^{i}\right\|^{2}}{N \min _{i, j}\left\|v^{j}-v^{i}\right\|^{2}}
$$

\section{Parameter estimation}

In this section, optimal number of rule-base Fuzzy partition matrix obtained by GK algorithm is used to estimate the premise parameters. Here, the Gaussian Membership Function (GMF) for premise variables is considered. The mean and the width (standard deviation) [38], [31], [32], [39], [40] are obtained as follows,

$$
\vartheta_{\mathrm{j}}{ }^{i}=\frac{\sum_{k=1}^{N} \mu_{i k}{ }^{m} \mathrm{x}_{k j}}{\sum_{k=1}^{N} \mu_{i k}{ }^{m}}
$$

$$
\sigma_{j}{ }^{i}=\sqrt{\frac{2 *\left[\sum_{k=1}^{N} \mu_{i k}{ }^{m}\left(x_{k j}-\vartheta_{j}{ }^{i}\right)^{2}\right]}{\sum_{k=1}^{N} \mu_{i k}{ }^{m}}}
$$

The premise model parameters are identified from the Fuzzy partition matrix $(U)$ and model data $(Z)$. The coefficients of consequent part are identified. The obtained premise parameters are applied to make a global matrix form of consequent coefficients. The matrix form of coefficients is expressed as,

$$
Y=\pi \zeta+\varepsilon
$$

where $\pi$ is the regression vector that is derived from the input vector and truth value $(w) . Y=\left[y_{1}, y_{2}, \ldots, y_{N}\right]$ is the observed data vector and $\varepsilon$ is the modeling error for $N^{t h}$ number of observations. The global form of the consequent coefficients is $\zeta=$ $\left[p_{0}{ }^{1}, p_{1}{ }^{1}, \ldots, p_{n}{ }^{1}, p_{0}{ }^{2}, p_{1}{ }^{2}, \ldots, p_{n}{ }^{2}, \ldots, p_{0}{ }^{C}, p_{1}{ }^{C}, \ldots, p_{n}{ }^{C}\right]$.

The regression vector is represented as,

$$
\pi\left(x_{k}\right)=\left[\begin{array}{lllllll}
\nabla_{1 k} & \nabla_{1 k} x_{k 1} & \nabla_{1 k} x_{k 2} \ldots \nabla_{1 k} x_{k n} & \ldots \nabla_{C k} & \ldots & \nabla_{C k} x_{k n}
\end{array}\right]
$$

where, the truth value is obtained as follows,

$$
\nabla_{i k}=\frac{w_{k}{ }^{i}}{\sum_{i=1}^{C} w_{k}{ }^{i}}
$$

The matrix inversion of Equation (19) may not feasible for all the time to determine the coefficients of $\zeta$ [31]. Therefore, the Orthogonal Least Square (OLS) has been applied to the Equation (19) to identify the coefficients of consequent parameters [32].

\section{Proposed TS Fuzzy Model}

The selection of input and output variables is the first step to develop a TS-Fuzzy rainfall-runoff model. Runoff at the outlet of a drainage basin is directly related to previous values of runoff and rainfall at different locations. However, the accumulated water due to rainfall takes a variable time to reach the exit of the basin depending on the catchment topography. The time interval from the center of mass of rainfall excess to the peak of the resulting hydrograph is known as lag-time. The effect of lag-time has been removed from the output dataset by adding the same delay in all the input variables. We define our proposed model after (removing the lag-time in the training dataset) as $M_{4}$ model (with four input variable), having the following data structure:

$$
M_{4}: \quad y_{k}=f\left(y_{k-1}, x_{k}^{1}, x_{k}^{2}, x_{k}^{3}\right)
$$

where $y_{k}$ is the observed runoff and $x_{k}^{1}, x_{k}^{2}$ and $x_{k}^{3}$ are the rainfall collected at different locations at the $k^{\text {th }}$ time interval of a time horizon $0, T$ hours. Let $\delta_{\text {pred }}^{0}=$ time difference between $k^{\text {th }}$ and $k-1^{\text {th }}$ data point. We define the set $\delta_{\text {pred }}=\delta_{\text {pred }}^{0}, \ldots, \delta_{\text {pred }}^{i_{N}}, \ldots$ such that $\delta_{\text {pred }}^{i_{N}-1}=i_{N} \cdot \delta_{\text {pred }}^{0}$ and so on, where $i_{N}$ is an integer. A subset of $\delta_{\text {pred }}$ is called as a time ahead runoff prediction scheme. 


\section{A. Flow chart of a GK algorithm based TS Fuzzy model}

Figure 1 describes the general architecture of the proposed GK algorithm based TS Fuzzy model. The model architecture is broadly classified into the following:

a) Finding optimal rule-base: The GK clustering algorithm (Algorithm1) along with validation index has been applied to input-output data set to create optimal Fuzzy subspaces (Fuzzy rules). Each optimal Fuzzy subspace is associated to a Fuzzy rule i.e. the output of optimal Fuzzy ubspaces is described by Fuzzy partition matrix $(U)$. Once the optimal subspace is obtained from the both algorithms, then obtained $U$ has been used to estimate the model parameters.:

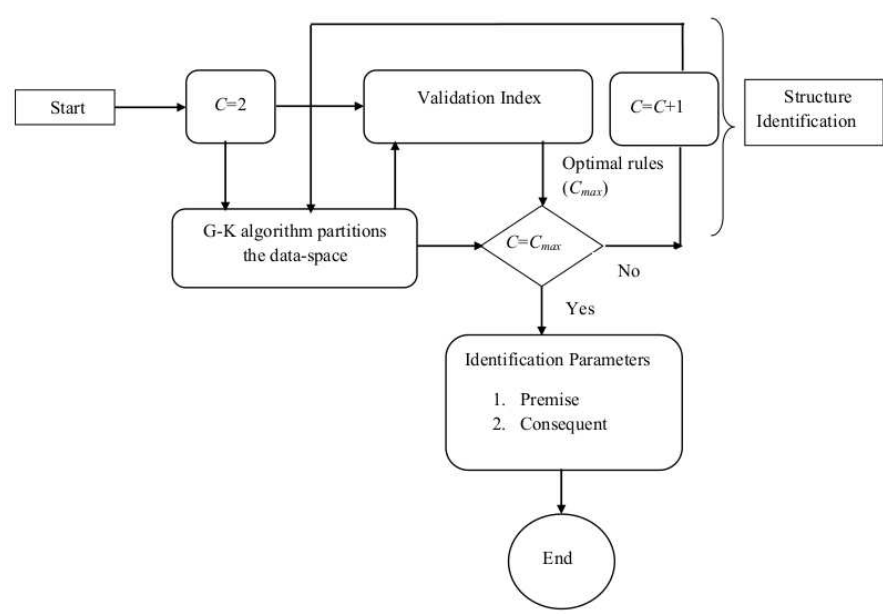

Fig. 1. Architecture of the GK algorithm based TS Fuzzy model

b) Estimating TS Fuzzy model parameters: Gaussian membership function parameters (mean and width) are obtained by using eq.(17) and eq.(18) respectively. We have formulated a global matrix form of regression vector to identify the consequent parameters. OLS is applied to eq.(19) estimate the consequent parameters.:

\section{Data And validation technique}

A part of the campus of IIT Kharagpur is used as a pilot test-bed for the data acquisition process as shown in Figure 2. The validation data are obtained from an real-time web-based hydro-meteorological sensor network platform that exists in the study area [10]. In Figure 2, the red markers are indicating locations of tipping bucket rain-gauges for on-line measurement of rainfall, and the blue up arrow indicates outlet of the drainage basin, where a rectangular weir with pressure type digital water depth sensor is installed to calculate pressure head (or water level). Pressure head is converted to surface runoff flow using Equation 1, 2 and 3 of [10].

A rainfall-runoff data set collected from the storm event in the $05^{\text {th }}$ May, 2015 between 21: 12 Hours to $23: 52$ Hours, comprising of 302 observations (Data Set 1) is used to train the model parameters. Collected pressure head data from the storm event in the $22^{\text {nd }}$ September, 2015 between 21:40 Hours to $23: 40$ Hours, comprising of 273 observations (Data

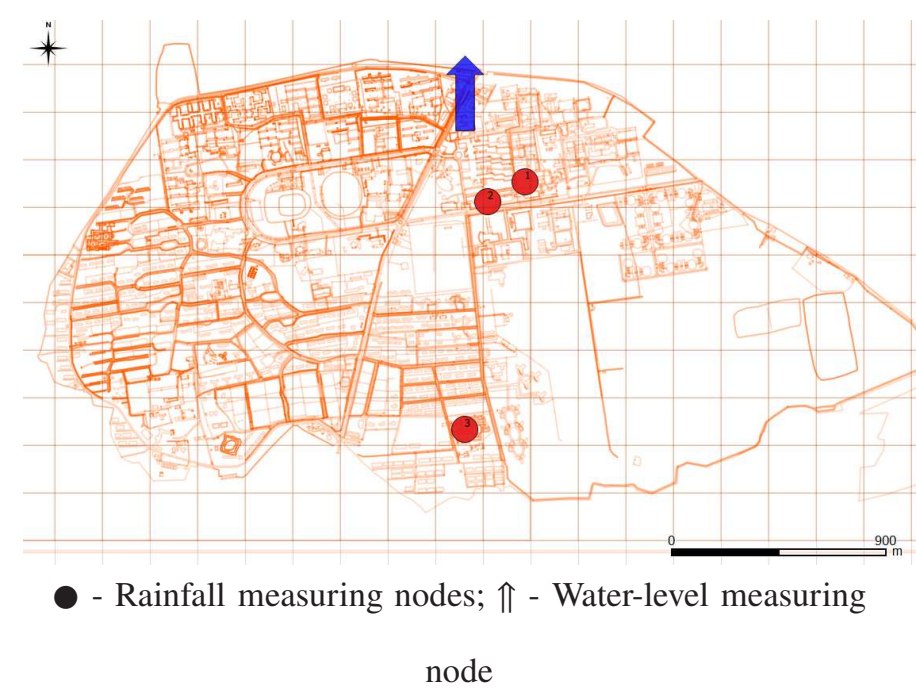

Fig. 2. Actual locations of spatially distributed sensing nodes deployed in the pilot study area

Set 2) are used to validate the models. Each observation used for training and validation purpose is comprises of rainfall data obtained from three spatially distributed location and pressure head data obtained at the exit of the study area. In Data set 1 (training data) and Data set 2 (validation data), collected data are taken at 30 seconds interval. Hence, the minimum time difference between each observation $\delta_{\text {pred }}^{0}$ is 30 seconds in Data Set 1 and Data Set 2. The proposed model is trained with Data Set 1 and validated using Data Set 2 without changing the dimension of each data point. Next, we normalise the input output dataset to develop a dimensionless training and validation data set. The models are labelled with a parenthesis $(N)$ when dimensionless input and output data set used to calculate the performance measure. The model performance is validated through statistical and hydrological performance measures such as Root Mean Square Error (RMSE), Coefficient of Efficiency (CE), Volumetric Error (VE) and correlation coefficient of determination (R) between observed data and simulated data. The model performance criteria are given by,

$$
\begin{aligned}
& R M S E=\sqrt[2]{\frac{\sum_{k=1}^{N}\left(y_{k}-\hat{y}_{k}\right)^{2}}{N}} \\
& C E=\left(1-\frac{F}{F_{0}}\right) ; \\
& V E=\left(\frac{\sum y_{k}-\sum \hat{y}_{k}}{\sum y_{k}}\right) \times 100 \\
& R=\frac{\sum_{k=1}^{N}\left(y_{k}-\bar{y}\right)\left(\hat{y}_{k}-\bar{y}_{p}\right)}{\sqrt[2]{\sum_{k=1}^{N}\left(y_{k}-\bar{y}\right)^{2}} \sqrt[2]{\sum_{k=1}^{N}\left(\hat{y}_{k}-\bar{y}_{p}\right)^{2}}} ; \bar{y}_{p}=\frac{1}{N} \sum_{k=1}^{N} \hat{y}_{k}
\end{aligned}
$$

where $y_{k}$ is the observed pressure head (in $\mathrm{mm}$ ), $\hat{y}_{k}$ is the predicted pressure head from the model. $\bar{y}$ and $\bar{y}_{p}$ are the mean of observed and predicted data respectively. The RMSE performance measure shows the algorithm capability for predicting the observed data. Lower values of RMSE are indicating better fit with the observed data. The $C E$ performance measure indicates the quality of the model verification data taken for different lengths over different time interval. The correlation 
TABLE I. Prediction error comparison for all the different algorithm based $M_{4}$ model in both dimensional and dimensionless input-output setting

\begin{tabular}{|c|c|c|c|c|c|c|c|c|}
\hline & \multicolumn{4}{|c|}{ Training } & \multicolumn{4}{|c|}{ Validation } \\
\hline Algorithm & RMSE & VE & $\mathrm{CE}$ & $\mathrm{R}$ & RMSE & VE & $\mathrm{CE}$ & $\mathrm{R}$ \\
\hline$G K$ & 0.00 & 0.00 & 1.0 & 1.00 & 0.00 & 0.00 & 1.0 & 1.0 \\
\hline$G K(N)$ & 0.00 & 0.002 & 0.99 & 0.99 & 0.00 & 0.002 & 0.99 & 1 \\
\hline$\overline{F C M}$ & 0.04 & 0.013 & 1 & 1 & 0.49 & 0.05 & 1 & 1 \\
\hline$F C M(N)$ & 0.02 & 1.09 & 0.99 & 1 & 0.02 & 2.29 & 0.99 & 1 \\
\hline$S C$ & 0.03 & 0.01 & 1 & 1 & 0.06 & 0.01 & 1 & 1 \\
\hline$S C(N)$ & 0.01 & 1.12 & 1 & 1 & 0.04 & 2.96 & 0.98 & 0.99 \\
\hline
\end{tabular}

coefficient $(R)$ is used to check the goodness-of-fit of the model.

\section{Results And Discussion}

We discuss two types of errors: (i) prediction error i.e. error observed in the next immediate observation, and (ii) time ahead runoff prediction error i.e. error observed in a particular time ahead runoff prediction scheme.

\section{A. Prediction error comparison}

GK, FCM and SC algorithms based $M_{4}$ TS Fuzzy models are constructed for both training (Data Set 1) and validation (Data Set 2). Table I, specifies the performances of $M_{4}$. The performance of the $M_{4}$ model is listed in the Table I for both dimensional and dimensionless input of the Data Set 2. The performances of six different cluster validation indices as presented in Section IV-C have given minimum values at 3 clusters. Hence, there are 3 optimal number of clusters (rules) present in the model data set of $M_{4}$. Error measures for the six M4 models are presented in Table I for both the training and validation data. In Table I, the row that contains dimensionless input are identifiable by the ' $(\mathrm{N})$ ' beside the algorithm (e.g. $G K(N))$.

The FCM algorithm based $M_{4}$ model is generated by MATLAB "genfis3" function. The optimal number of rules in the FCM based Fuzzy structure are similar to the optimal number of rules, obtained by GK validation indices. Table I indicates that FCM algorithm based model is not as good as GK algorithm while estimating the runoff data. The SC algorithm based $M_{4}$ model is generated by MATLAB "genfis2" function where number of rules in the model structure is similar to the optimal number of rules (provided by GK validation indices). Table I indicates that SC based TS Fuzzy model has a better performance than that of FCM algorithm but it is not as good as the GK algorithm based $M_{4}$ model.

The GK based $M_{4}$ model performances for the training dataset and validation dataset are shown in the Figure 3 and Figure 4 . The training and testing dataset performances by SC based $M_{4}$ model are shown in the Figure 7 and Figure 8 respectively. The FCM based $M_{4}$ model performances for the training and testing dataset are shown in the Figure 5 and Figure 6 respectively.

\section{B. Time ahead runoff prediction error comparison}

This section presents an error performance comparison of the algorithmic performances of the $M_{4}$ model when the
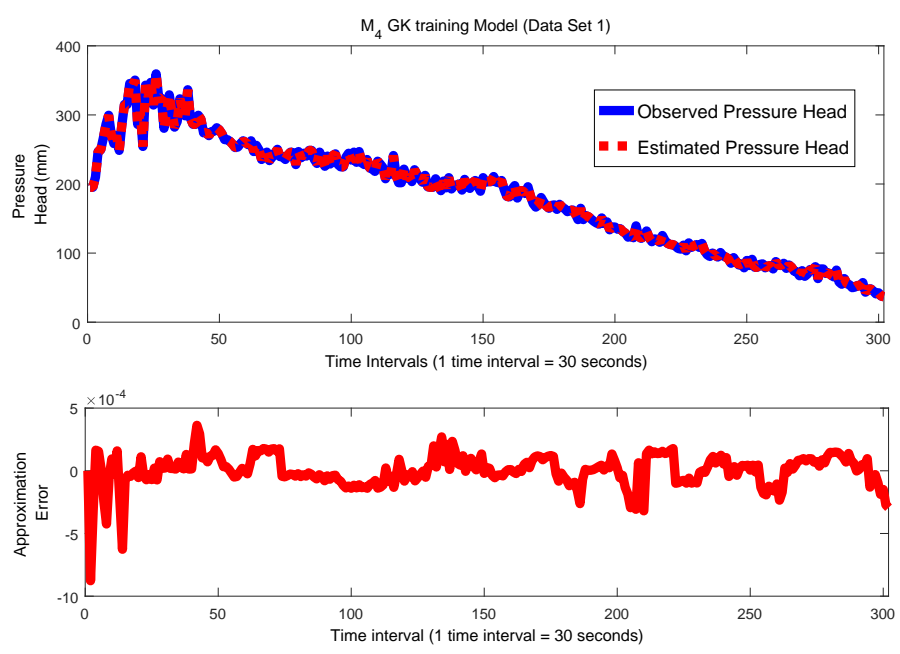

Fig. 3. Observed vs estimated pressure head for GK based $M_{4}$ model
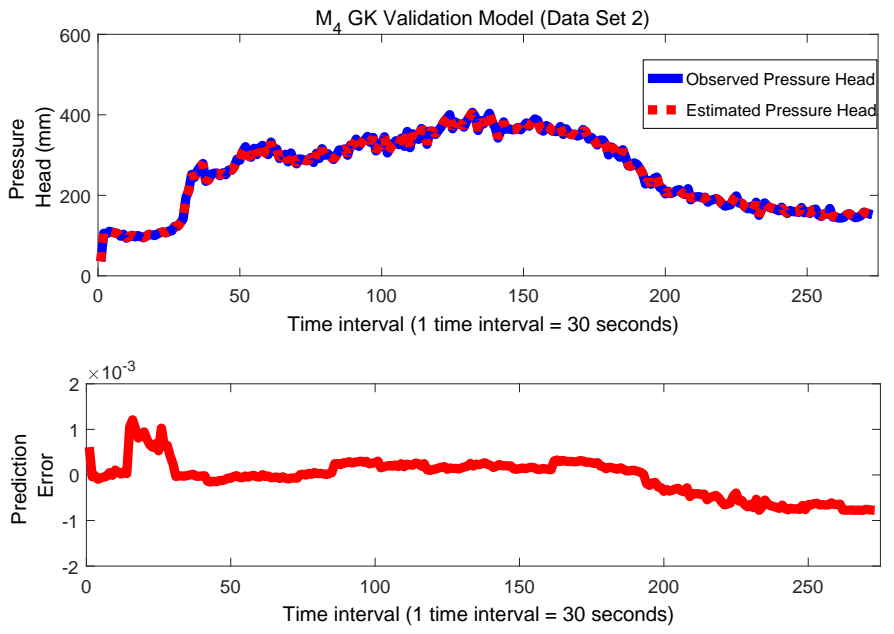

Fig. 4. Observed vs estimated pressure head for GK based $M_{4}$ model
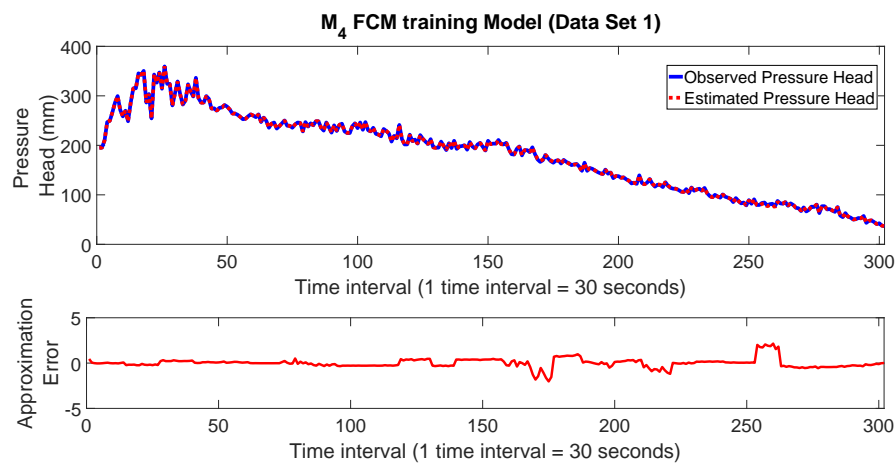

Fig. 5. Observed vs estimated pressure head for FCM based $M_{4}$ model

data is predicted according to three different prediction ahead time i.e. three different schemes selected from the set $\delta_{\text {pred }}$. We demonstrate a comparison between $\delta_{\text {pred }}^{1}=60$ seconds, 

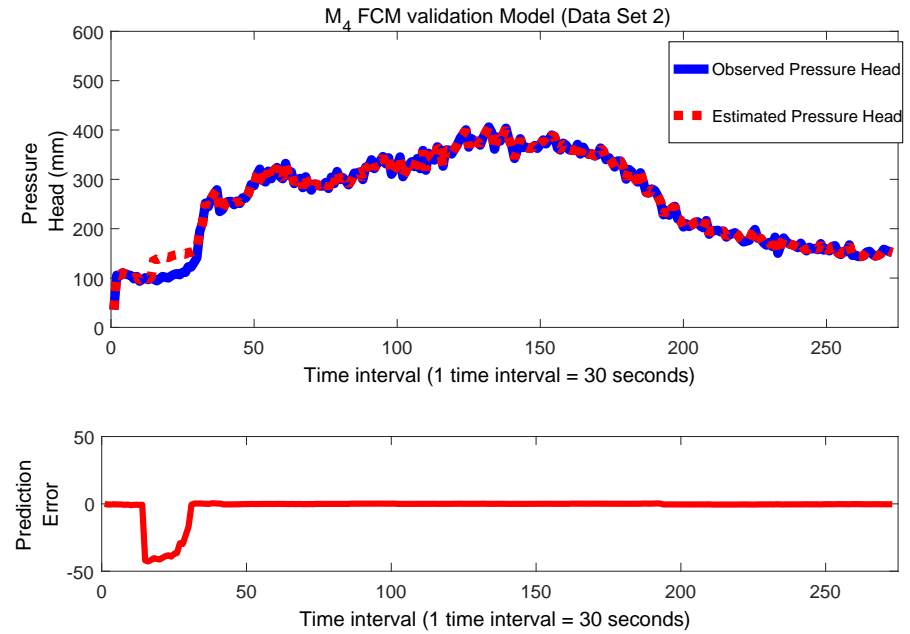

Fig. 6. Observed vs estimated pressure head for FCM based $M_{4}$ model
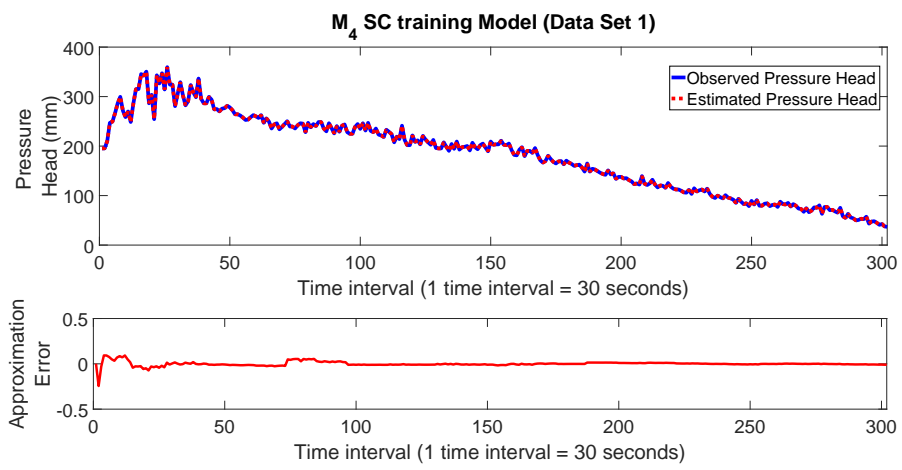

Fig. 7. Observed vs estimated pressure head for SC based $M_{4}$ model
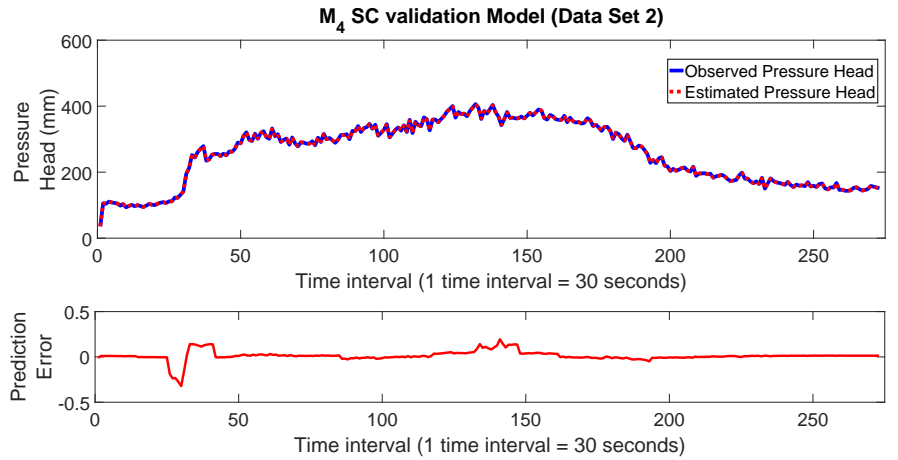

Fig. 8. Observed vs estimated pressure head for SC based $M_{4}$ model

$\delta_{\text {pred }}^{4}=150$ seconds and $\delta_{\text {pred }}^{9}=300$ seconds in Table II. We should note that Table I represents the errors obtained in the time ahead prediction scheme $\delta_{\text {pred }}^{0}=30$ seconds.

Table II shows that the RMSE error of the $M_{4}$ model for GK algorithm has a $\pm 16 \%$ tolerance while predicting the runoff 10 times (300s/30s) earlier. The RMSE error of the $M_{4}$ model for FCM and SC algorithms have a $\pm 18 \%$ tolerance for the same prediction time interval. Figure 9 is showing the estimated
TABLE II. Error comparison for three different prediction ahead time

\begin{tabular}{l|llll|lllll|}
\multirow{2}{*}{$\delta_{\text {pred }}^{1}$} & \multicolumn{5}{|c|}{ Training } & \multicolumn{5}{c|}{ Validation } \\
& RMSE & VE & CE & R & RMSE & VE & CE & $\mathrm{R}$ \\
\hline$G K$ & 0.00 & 0.00 & 1 & 1 & 8.99 & 0.3 & 0.99 & 0.99 \\
$F C M$ & 0.93 & 0.35 & 0.99 & 0.99 & 9.42 & 1.07 & 0.99 & 0.99 \\
$S C$ & 0.33 & 0.05 & 1 & 1 & 9.25 & 0.31 & 0.99 & 0.99 \\
\hline \hline$\delta_{\text {pred }}^{4}$ & \multicolumn{5}{|c|}{ Training } & \multicolumn{5}{c|}{ Validation } \\
& RMSE & VE & CE & R & RMSE & VE & CE & $\mathrm{R}$ \\
\hline$G K$ & 0.00 & 0.00 & 1 & 1 & 12 & 0.61 & 0.99 & 0.99 \\
$F C M$ & 0.26 & 0.11 & 1 & 1 & 13.01 & 0.88 & 0.98 & 0.99 \\
$S C$ & 0.07 & 0.02 & 1 & 1 & 12.94 & 0.63 & 0.98 & 0.99 \\
\hline \hline$\delta_{\text {pred }}^{9}$ & \multicolumn{5}{|c|}{ Training } & & \multicolumn{5}{c|}{ Validation } \\
& RMSE & VE & CE & R & RMSE & VE & CE & $\mathrm{R}$ \\
\hline$G K$ & 0.00 & 0.00 & 1 & 1 & 16.05 & 1.12 & 0.97 & 0.99 \\
$F C M$ & 0.014 & 0.004 & 1 & 1 & 18.06 & 1.23 & 0.96 & 0.99 \\
$S C$ & 0.006 & 0.002 & 1 & 1 & 18.05 & 1.21 & 0.96 & 0.99 \\
\hline
\end{tabular}

pressure head before 5 minutes of observed runoff for FCM based $M_{4}$ model that has the highest RMSE error among rest of the models.

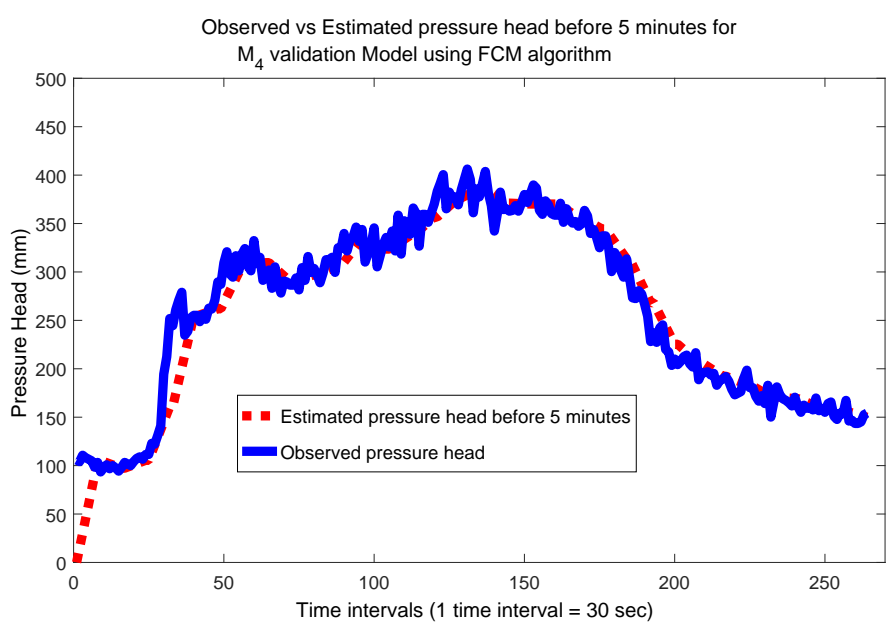

Fig. 9. Estimated pressure head before 5 minutes of observed runoff for FCM based $M_{4}$ model

\section{Conclusion And Future Work}

This paper presents a GK clustering algorithm based TS fuzzy model to predict runoff due to rainfall using read world observations. Further, a comparative study between FCM and SC clustering algorithms is developed with various error measures. The proposed model is trained and validated with a different set of observations recorded on different days. Validation results show that the GK algorithm based $M_{4}$ model performs significantly better than FCM and SC algorithms. GK algorithm also performs better in the time ahead runoff prediction error that increases with $i_{N}$ (the superscript of $\delta_{\text {pred }}^{i_{N}}$.

Proposed models have been trained and validated over a limited number of rainfall-runoff data due to the catchment geography and climate characteristics. As future work, the methodologies described in this paper may be applied to those 
catchments where rainfall-runoff data is available for a longer duration of time to predict the surface runoff due to rainfall. Other hydrological inputs (e.g. Antecedent moisture conditions) and/or an increasing number of spatially distributed rainfall stations may be integrated with the input data set for a better prediction of the output.

\section{REFERENCES}

[1] S. N. Kuiry, D. Sen, and P. D. Bates, "Coupled 1d-quasi-2d flood inundation model with unstructured grids," Journal of Hydraulic Engineering, vol. 136, no. 8, pp. 493-506, 2010.

[2] A. P. Jacquin and A. Y. Shamseldin, "Development of rainfall-runoff models using takagi-sugeno fuzzy inference systems," Journal of Hydrology, vol. 329, no. 1-2, pp. 154-173, 2006.

[3] Y. Morales, M. Querales, H. Rosas, H. Allende-Cid, and R. Salas, "A self-identification neuro-fuzzy inference framework for modeling rainfall-runoff in a chilean watershed," Journal of Hydrology, vol. 594, p. 125910, 2021.

[4] A. K. Lohani, N. Goel, and K. Bhatia, "Development of fuzzy logic based real time flood forecasting system for river narmada in central india," in International conference on innovation advances and implementation of flood forecasting technology, vol. 17, 2005, pp. 1-10.

[5] A. Lohani, N. Goel, and K. Bhatia, "Takagi-sugeno fuzzy inference system for modeling stage-discharge relationship," Journal of Hydrology, vol. 331, no. 1, pp. 146-160, 2006.

[6] Y. Hundecha, A. Bardossy, and H.-W. WERNER, "Development of a fuzzy logic-based rainfall-runoff model," Hydrological Sciences Journal, vol. 46, no. 3, pp. 363-376, 2001.

[7] J. shing Roger Jang, "Anfis: Adaptive-network-based fuzzy inference system," IEEE Transactions on Systems, Man, and Cybernetics, vol. 23, pp. 665-685, 1993.

[8] T. K. Chang, A. Talei, S. Alaghmand, and M. P.-L. Ooi, "Choice of rainfall inputs for event-based rainfall-runoff modeling in a catchment with multiple rainfall stations using data-driven techniques," Journal of Hydrology, vol. 545, pp. 100-108, 2017.

[9] R. Tabbussum and A. Q. Dar, "Performance evaluation of artificial intelligence paradigms - artificial neural networks, fuzzy logic, and adaptive neuro-fuzzy inference system for flood prediction," Environmental Science and Pollution Research, vol. 28, no. 20, pp. $25265-$ $25282,2021$.

[10] S. Dey, N. Bhattacharya, S. Chakrabarti, and D. Sen, "Real-time ogc compliant online data monitoring and acquisition network for management of hydro-meteorological hazards," ISH Journal of Hydraulic Engineering, pp. 1-10, 2016.

[11] A. K. Lohani, N. Goel, and K. Bhatia, "Comparative study of neural network, fuzzy logic and linear transfer function techniques in daily rainfall-runoff modelling under different input domains," Hydrological Processes, vol. 25, no. 2, pp. 175-193, 2011.

[12] C. W. Dawson and R. Wilby, "An artificial neural network approach to rainfall-runoff modelling," Hydrological Sciences Journal, vol. 43, no. 1, pp. 47-66, 1998.

[13] G. Tayfur and V. P. Singh, "Ann and fuzzy logic models for simulating event-based rainfall-runoff," Journal of hydraulic engineering, vol. 132, no. 12, pp. 1321-1330, 2006.

[14] F.-J. Chang and Y.-C. Chen, "A counterpropagation fuzzy-neural network modeling approach to real time streamflow prediction," J. Hydrol., vol. 245 , no. $1-4$, pp. 153-164, 2001.

[15] P. Nayak, K. Sudheer, and K. Ramasastri, "Fuzzy computing based rainfall-runoff model for real time flood forecasting," Hydrological Processes, vol. 19, no. 4, pp. 955-968, 2005.

[16] P. C. Nayak, K. Sudheer, D. Rangan, and K. Ramasastri, "A neuro-fuzzy computing technique for modeling hydrological time series," Journal of Hydrology, vol. 291, no. 1, pp. 52-66, 2004.

[17] P. Nayak, K. Sudheer, D. Rangan, and K. Ramasastri, "Short-term flood forecasting with a neurofuzzy model," Water Resources Research, vol. 41, no. 4, 2005.

[18] A. Mukerji, C. Chatterjee, and N. S. Raghuwanshi, "Flood forecasting using ann, neuro-fuzzy, and neuro-ga models," Journal of Hydrologic Engineering, vol. 14, no. 6, pp. 647-652, 2009.

[19] R. Remesan, M. A. Shamim, D. Han, and J. Mathew, "Runoff prediction using an integrated hybrid modelling scheme," Journal of Hydrology, vol. 372 , no. 1, pp. 48-60, 2009.
[20] N. K. Kasabov and Q. Song, "Denfis: dynamic evolving neural-fuzzy inference system and its application for time-series prediction," IEEE transactions on Fuzzy Systems, vol. 10, no. 2, pp. 144-154, 2002.

[21] S. A. Akrami, V. Nourani, and S. Hakim, "Development of nonlinear model based on wavelet-anfis for rainfall forecasting at klang gates dam," Water resources management, vol. 28, no. 10, pp. 2999-3018, 2014.

[22] A. Talei and L. H. Chua, "Influence of lag time on event-based rainfallrunoff modeling using the data driven approach," Journal of hydrology, vol. 438, pp. 223-233, 2012.

[23] R. Govindraju, "Artificial neural networks in hydrology: Ii. hydrological applications," J. Hydrol. Eng, vol. 5, no. 2, pp. 124-137, 2000.

[24] L. H. Chua, T. S. Wong, and L. Sriramula, "Comparison between kinematic wave and artificial neural network models in event-based runoff simulation for an overland plane," Journal of hydrology, vol. 357, no. 3, pp. 337-348, 2008.

[25] A. Talei, L. H. C. Chua, and C. Quek, "A novel application of a neurofuzzy computational technique in event-based rainfall-runoff modeling," Expert Systems with Applications, vol. 37, no. 12, pp. 7456-7468, 2010.

[26] A. Talei, L. H. C. Chua, C. Quek, and P.-E. Jansson, "Runoff forecasting using a takagi-sugeno neuro-fuzzy model with online learning," Journal of hydrology, vol. 488, pp. 17-32, 2013.

[27] P. Nayak, K. Sudheer, and S. Jain, "Rainfall-runoff modeling through hybrid intelligent system," Water Resources Research, vol. 43, no. 7, 2007.

[28] T. Takagi and M. Sugeno, "Fuzzy identification of systems and its applications to modeling and control," IEEE transactions on systems, man, and cybernetics, no. 1, pp. 116-132, 1985.

[29] S. L. Chiu, "Fuzzy model identification based on cluster estimation," Journal of Intelligent \& fuzzy systems, vol. 2, no. 3, pp. 267-278, 1994.

[30] J. Abonyi, R. Babuska, and F. Szeifert, "Modified gath-geva fuzzy clustering for identification of takagi-sugeno fuzzy models," IEEE Transactions on Systems, Man, and Cybernetics, Part B (Cybernetics), vol. 32, no. 5, pp. 612-621, 2002

[31] T. Dam and A. K. Deb, "Interval type-2 modified fuzzy c-regression model clustering algorithm in ts fuzzy model identification," in Fuzzy Systems (FUZZ-IEEE), 2016 IEEE International Conference on. IEEE, 2016, pp. 1671-1676.

[32] _ - "Ts fuzzy model identification by a novel objective function based fuzzy clustering algorithm," in Computational Intelligence in Ensemble Learning (CIEL), 2014 IEEE Symposium on. IEEE, 2014, pp. 1-7.

[33] R. Babuka, P. Van der Veen, and U. Kaymak, "Improved covariance estimation for gustafson-kessel clustering," in Fuzzy Systems, 2002. FUZZ-IEEE'02. Proceedings of the 2002 IEEE International Conference on, vol. 2. IEEE, 2002, pp. 1081-1085.

[34] X. L. Xie and G. Beni, "A validity measure for fuzzy clustering," IEEE Transactions on pattern analysis and machine intelligence, vol. 13, no. 8, pp. 841-847, 1991.

[35] J. C. Bezdek, "Numerical taxonomy with fuzzy sets," Journal of Mathematical Biology, vol. 1, no. 1, pp. 57-71, 1974.

[36] R. N. Dave, "Validating fuzzy partitions obtained through c-shells clustering," Pattern Recognition Letters, vol. 17, no. 6, pp. 613-623, 1996.

[37] A. M. Bensaid, L. O. Hall, J. C. Bezdek, L. P. Clarke, M. L. Silbiger, J. A. Arrington, and R. F. Murtagh, "Validity-guided (re) clustering with applications to image segmentation," IEEE Transactions on Fuzzy Systems, vol. 4, no. 2, pp. 112-123, 1996.

[38] T. Dam and A. K. Deb, "A clustering algorithm based ts fuzzy model for tracking dynamical system data," Journal of the Franklin Institute, vol. 354, no. 13, pp. 5617-5645, 2017.

[39] T. Dam and A. Deb, "Block sparse representations in modified fuzzy cregression model clustering algorithm for ts fuzzy model identification," in 2015 IEEE Symposium Series on Computational Intelligence. IEEE, 2015, pp. $1687-1694$.

[40] T. Dam and A. K. Deb, "Interval type-2 recursive fuzzy c-means clustering algorithm in the ts fuzzy model identification," in 2015 IEEE Symposium Series on Computational Intelligence. IEEE, 2015, pp. 22-29. 


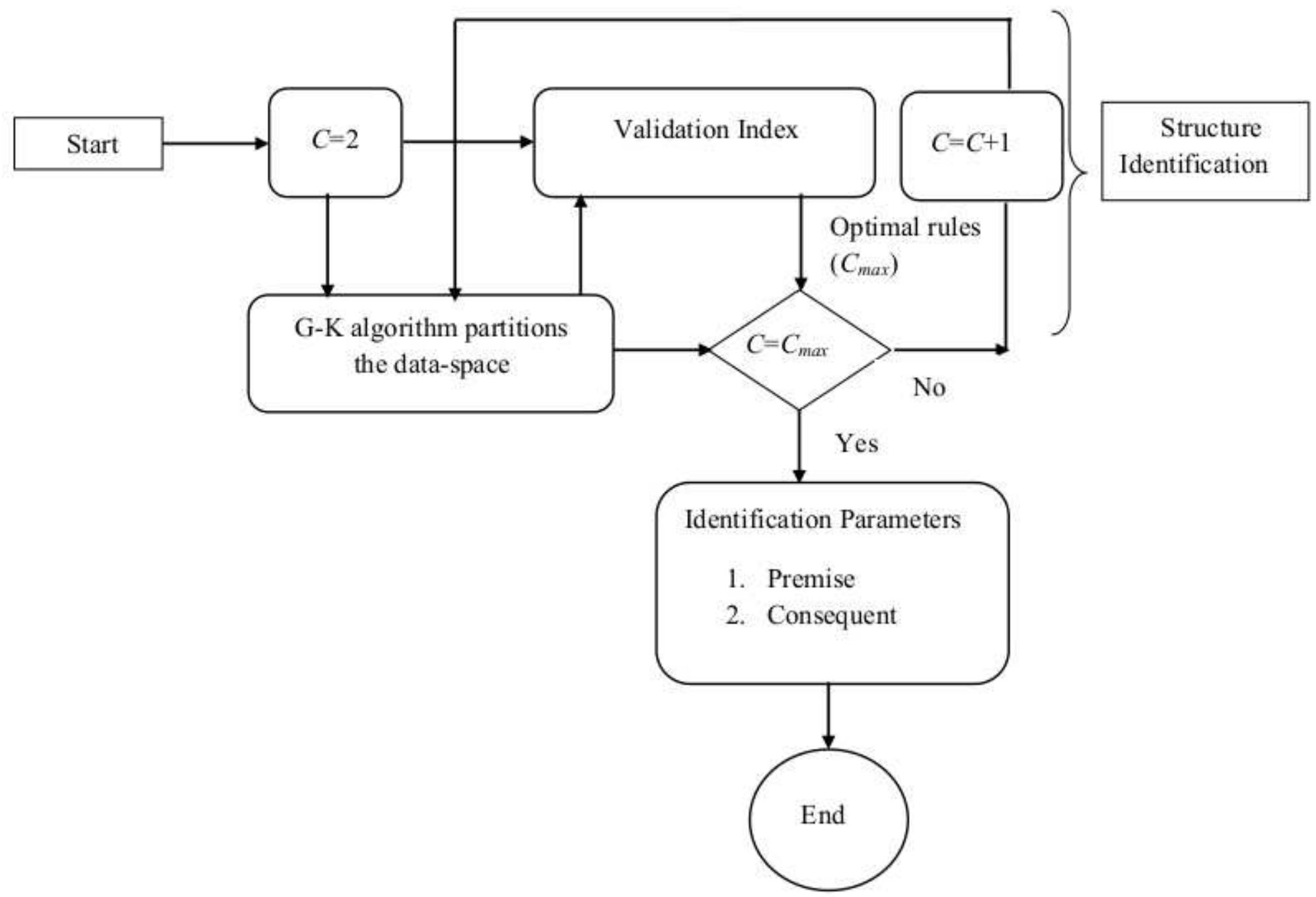

